\title{
Expression patterns of immune checkpoints in acute myeloid leukemia
}

\author{
Cunte Chen ${ }^{1+} \mathbb{D}$, Chaofeng Liang ${ }^{1+}$, Shunqing Wang ${ }^{2}$, Chi Leong Chio ${ }^{1}$, Yuping Zhang ${ }^{2}$, Chengwu Zeng ${ }^{1}$, \\ Shaohua Chen ${ }^{1^{*}}$, Caixia Wang ${ }^{2^{*}}$ and Yangqiu $\mathrm{Li}^{1^{*}}$ (i)
}

\begin{abstract}
Immunotherapy with immune checkpoint inhibitors (ICIs) for solid tumors had significantly improved overall survival. This type of therapy is still not available for acute myeloid leukemia (AML). One major issue is the lack of knowledge for the expression patterns of immune checkpoints (IC) in AML. In this study, we first explored the prognostic value of ICs for AML patients by analyzing RNA-seq and mutation data from 176 AML patients from the Cancer Genome Atlas (TCGA) database. We further validated the results of the database analysis by analyzing bone marrow (BM) samples from 62 patients with de novo AML. Both TCGA data and validation results indicated that high expression of PD-1, PD-L1, and PD-L2 was associated with poor overall survival (OS) in AML patients. In addition, increased co-expression of PD-1/CTLA-4 or PD-L2/CTLA-4 correlated with poor OS in AML patients (3-year OS: TGCA data $30 \%$ vs $0 \%$ and $20 \%$ vs $0 \%$, validation group $57 \%$ vs $31 \%$ and $57 \%$ vs $33 \%$, respectively) $(P<0.05)$. Moreover, co-expression of PD-1/PD-L1, PD-1/PD-L1/PD-L2, and PD-1/LAG-3 was found to correlate with poor OS in AML patients with FLT3 ${ }^{\text {mut }}$, RUNX1 ${ }^{\text {mut }}$, and TET2 ${ }^{\text {mut }}$, respectively. In conclusion, high expression of ICs in the BM leukemia cells of AML patients correlated with poor outcome. The co-expression patterns of PD-1/CTLA-4, PD-L2/ CTLA-4, PD-1/PD-L1, PD-1/PD-L1/PD-L2, and PD-1/LAG-3 might be potential immune biomarkers for designing novel AML therapy.
\end{abstract}

Keywords: PD-1, PD-L1, PD-L2, Prognosis, Immune checkpoint, AML

To the Editor,

Immune checkpoint (IC) blockade by inhibitors of the programmed cell death 1 (PD-1) and PD-1 ligand 1 (PD-L1) has significantly improved clinical outcome for a variety of solid tumors [1, 2], while little is known about the role of ICs in leukemia [3]. Previous reports have shown that higher numbers of PD-1 + T cells are related to poor outcome for patients with

\footnotetext{
*Correspondence: jnshaohuachen@163.com; wangcx225@163.com; yangqiuli@hotmail.com

${ }^{\dagger}$ Cunte Chen and Chaofeng Liang contributed equally to this work. 'Institute of Hematology, School of Medicine, Key Laboratory for

Regenerative Medicine of Ministry of Education, Jinan University, Guangzhou 510632, People's Republic of China

${ }^{2}$ Department of Hematology, Guangzhou First People's Hospital, School of Medicine, South China University of Technology, Guangzhou 510180,

People's Republic of China
}

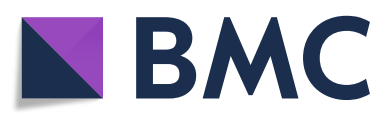

(c) The Author(s). 2020 Open Access This article is licensed under a Creative Commons Attribution 4.0 International License, which permits use, sharing, adaptation, distribution and reproduction in any medium or format, as long as you give appropriate credit to the original author(s) and the source, provide a link to the Creative Commons licence, and indicate if changes were made. The images or other third party material in this article are included in the article's Creative Commons licence, unless indicated otherwise in a credit line to the material. If material is not included in the article's Creative Commons licence and your intended use is not permitted by statutory regulation or exceeds the permitted use, you will need to obtain permission directly from the copyright holder. To view a copy of this licence, visit http://creativecommons.org/licenses/by/4.0/. The Creative Commons Public Domain Dedication waiver (http://creativecommons.org/publicdomain/zero/1.0/) applies to the data made available in this article, unless otherwise stated in a credit line to the data. using PD-1 inhibitors are ongoing to treat patients with a high risk for AML relapse [4]. However, the response rate varies widely, ranging from 22 to $72 \%$ [4], which may be due to heterogeneity in the IC expression level as well as distinct dominant IC expression patterns in different AML cases [5]. Therefore, it is worth studying the expression patterns of ICs in AML. In this study, we first explored the prognostic value of ICs in AML patients through analyzing RNA-seq and mutation data from the Cancer Genome Atlas (TCGA) database [6] and further validated the results by quantitative real-time PCR analysis of AML bone marrow (BM) samples from our clinical center. 


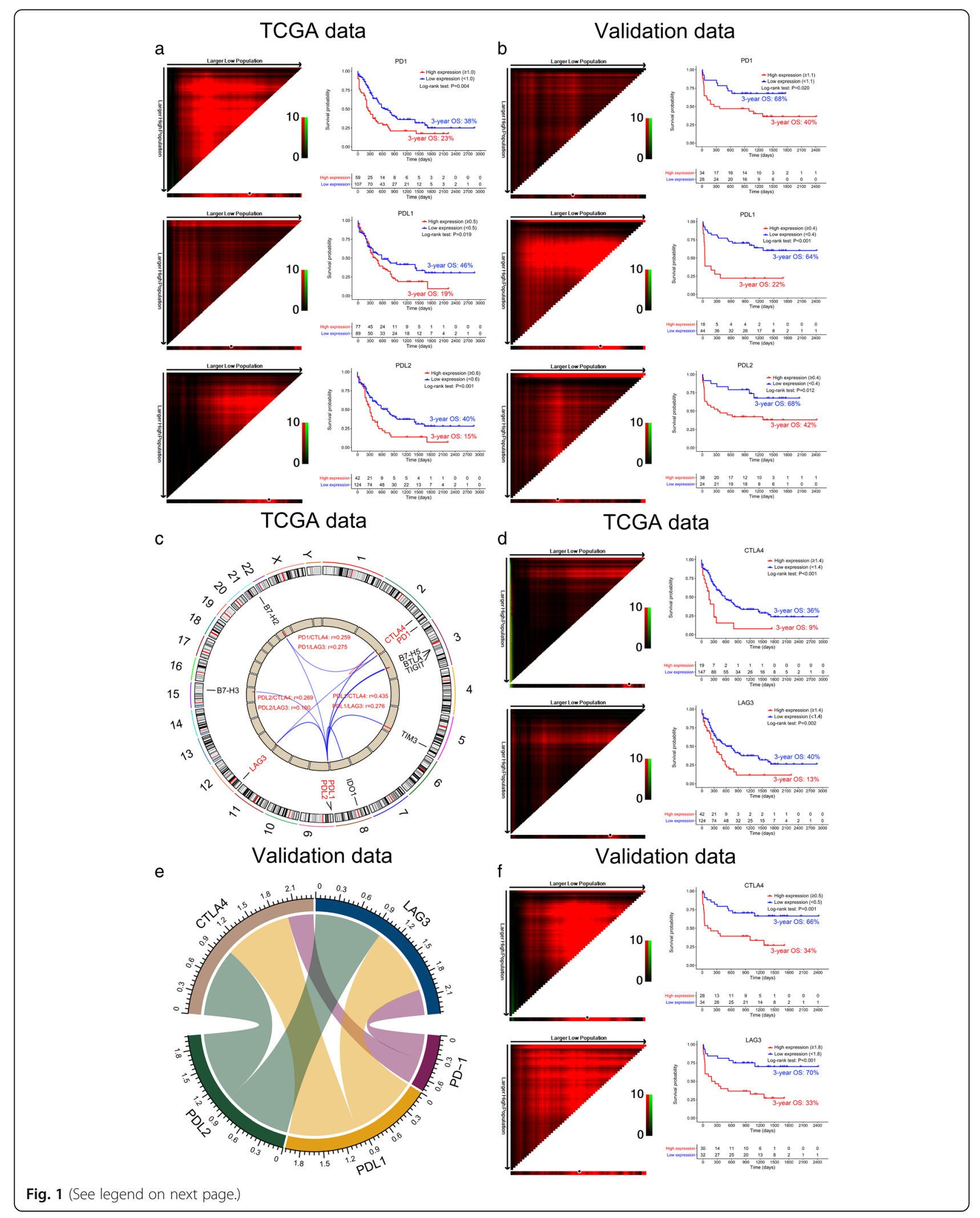


(See figure on previous page.)

Fig. 1 Overall survival (OS) of ICs in AML patients. a The OS probability in AML patients with high or low PD-1, PD-L1, or PD-L2 expression in TCGA group. (left panel) X-tile software (version 3.6.1) was used to define the optimal cutoff value for gene expression levels for prognosis, which is represented by the highest intensity pixel. Black dots represent the optimal cutoff value. The black to red or green in the color scale indicates that the range of pixels was from low to high. (right panel) Kaplan-Meier curves based on the optimal cutoff values. b The OS probability in AML patients with high or low PD-1, PD-L1, or PD-L2 expression in the validation group $(n=62)$. c Relationship between PD-1, PD-L1, and PD-L2 and other immune checkpoints in TCGA group. The outermost circle indicates 1 to 22, $\mathrm{X}$ and $\mathrm{Y}$ chromosomes; the second layer shows the location of the genes in the chromosomes; the third layer shows the IC genes; the innermost layer represents the average expression levels of the genes, which is shown by the height of the column; the lines in the center of the circle show the co-expression network of the PD-1, PD-L1, and PD-L2 and other ICs. The red font in the center of the circle displays the Pearson's coefficient with a $P$ value $<0.05$ for the correlation of two IC genes. d, $\mathbf{f}$ The OS probability in AML patients with high or low CTLA-4 and LAG-3 based on the optimal cutoff values provided by the X-tile software (version 3.6.1) in TCGA group (d) and in the validation group (f). e The chord diagram shows the co-expression network between PD-1, PD-L1, PD-L2, CTLA-4, and LAG-3 in BM samples from AML patients in the validation group $(n=62)$. The band represents a positive correlation between the two IC genes, and the thickness indicates the magnitude of the Pearson's correlation coefficient (the $P$ value for testing the correlation coefficient was $<0.05)$

A total of 176 de novo AML patients from the TCGA database and 62 AML BM samples were used for overall survival (OS) analysis and validation. Higher expression of PD-1, PD-L1, and PD-L2 correlated with poor OS in the TCGA database analysis (3year OS $23 \%$ vs $38 \%, 19 \%$ vs $46 \%$, and $15 \%$ vs $40 \%$, respectively, $P<0.05)$. This result was confirmed in the validation group (3-year OS $40 \%$ vs $68 \%, 22 \%$ vs $64 \%$, and $42 \%$ vs $68 \%$, respectively, $P<0.05$, Fig. $1 \mathrm{a}, \mathrm{b})$. We further analyzed the expression patterns of PD-1, PDL1, and PD-L2 with other important ICs [7-9]. Subsequently, with Pearson's correlation analysis, we found that the expression of PD-1, PD-L1, or PD-L2 was positively associated with the expression of cytotoxic T-lymphocyte associated protein 4 (CTLA-4) $(r=$ $0.259, P<0.001 ; r=0.435, P<0.001 ; r=0.269, P<$ 0.001 , respectively) and lymphocyte activation gene-3 (LAG-3) $(r=0.275, P<0.001 ; r=0.276, P<0.001 ; r=$ $0.160, P=0.033$, respectively) in the TCGA group (Fig. 1c). This concomitant expression pattern was again confirmed in the validation group (Fig. 1e), showing the possibility of concomitant expression of PD-1, PD-L1, or PD-L2 with CTLA-4 $(r=0.373, P=$ $0.003 ; r=0.998, P<0.001 ; r=0.998, P<0.001$, respectively) and LAG3 $(r=0.372, P=0.003 ; r=$ 0.994, $P<0.001 ; r=0.994, P<0.001$, respectively). AML patients with high expression of CTLA-4 and LAG-3 were found to have poor OS (3-year OS 9\% vs $36 \%$ and $13 \%$ vs $40 \%$ respectively) (Fig. 1d). This result was again confirmed in the validation group (Fig. 1f) (3-year OS: CTLA-4 34\% vs 66\%, LAG-3 33\% vs $70 \%)$.

Combination of IC inhibitors (ICIs) has the potential to improve responses $[4,10]$. We analyzed expression patterns of ICs and found that pairwise combinations of PD-1, PD-L1, and PD-L2 and CTLA-4 and LAG-3 correlated with poor OS in AML patients $(P<0.05$,
Figure S1). Furthermore, among AML patients with high expressions of PD-1 or PD-L2, concomitant high expression of CTLA-4 correlated with poor OS in both the TCGA database (3-year OS: PD- $1^{\text {high }}$ CTLA- $4^{\text {low }}$ vs PD$11^{\text {high }}$ CTLA $-4^{\text {high }} 30 \%$ vs $0 \%, 20 \%$ vs $0 \%$ ) and validation group (3-year OS: PD-L2 ${ }^{\text {high }}$ CTLA- $4{ }^{\text {low }}$ vs PDL $2^{\text {high }}$ CTLA- $4^{\text {high }} 57 \%$ vs $31 \%, 57 \%$ vs $\left.33 \%\right)(P<0.05$, Fig. 2a, b). AML with PD-L $1^{\text {high }}$ CTLA- $4^{\text {high }}$ correlated with poor OS in the TCGA dataset (3-year OS $24 \%$ vs $0 \%, P<0.001)$; however, OS was not significantly different in the validation group (3-year OS $33 \%$ vs $20 \%$, $P=0.353$, Fig. 2a, b). In addition, high expression of LAG-3 with PD-1 ${ }^{\text {high }}$, PD-L1 $1^{\text {high }}$, or PD-L2 $2^{\text {high }}$ failed to correlate with OS in the TCGA and validation groups (Figures S2A - B).

To obtain the effects of PD-1, PD-L1, and PD-L2 on the prognosis of AML patients with genetic mutations, we analyzed OS of the top ten AML patients with a recurrent mutation (Figures S3), including $\mathrm{FLT3}^{\mathrm{mut}}$, RUNX1 $^{\text {mut }}$, or TET2 ${ }^{\text {mut }}$ [11-13]. Interestingly, in comparison with AML patients without such mutations, high co-expressions of PD-1/PD-L1 $(P=0.029)$, PD-1/PD-L1/ PD-L2 $(P=0.003)$, and PD-1/LAG-3 $(P<0.001)$ were found to be associated with poor OS in AML patients with FLT3 ${ }^{\text {mut }}$, RUNX1 $^{\text {mut }}$, or TET2 ${ }^{\text {mut }}$ (1-year OS $0 \%$ vs $58 \%$ vs $49 \%, 0 \%$ vs $56 \%$ vs $100 \%$, and $0 \%$ vs $100 \%$ vs $63 \%$, respectively) (Fig. 2c).

To the best of our knowledge, we for the first time described that high co-expressions of PD-1/ CTLA-4 and PD-L2/CTLA-4 correlated with poor OS of AML patients. Moreover, high co-expressions of PD-1/PD-L1, PD-1/PD-L1/PD-L2, and PD-1/ LAG-3 were associated with poor OS of AML patients with $\mathrm{FLT}^{\mathrm{mut}}$, RUNX1 $1^{\text {mut }}$, or $\mathrm{TET} 2^{\text {mut }}$, respectively (Fig. 2d). These co-expression patterns might be potential immune biomarkers for designing novel AML therapy. 


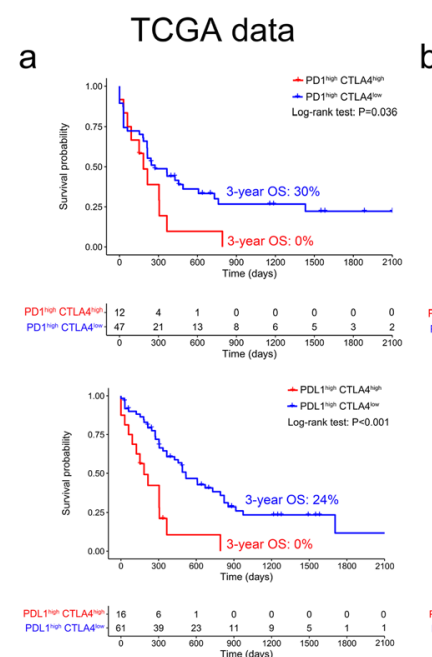

b
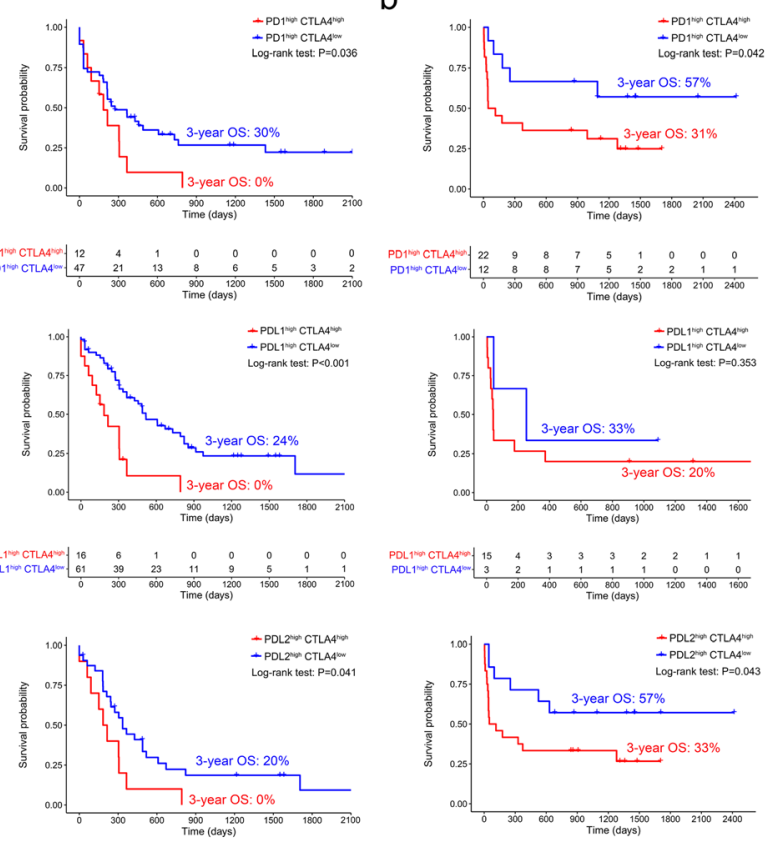

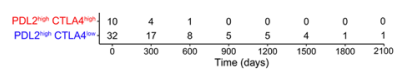

d
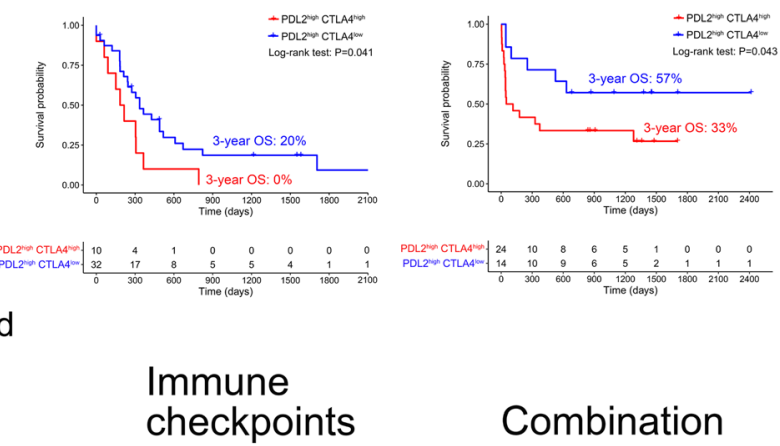

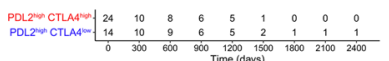

C

\section{TCGA data}
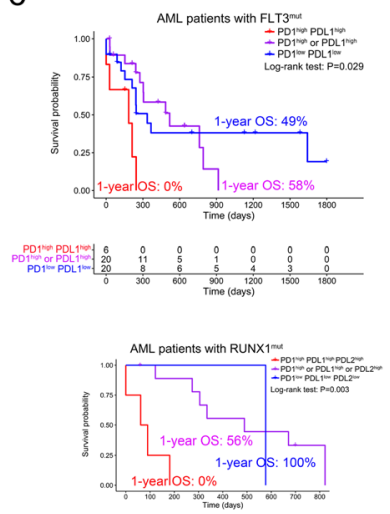

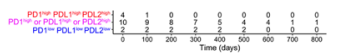

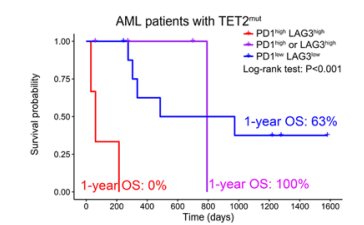

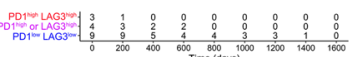

Patients

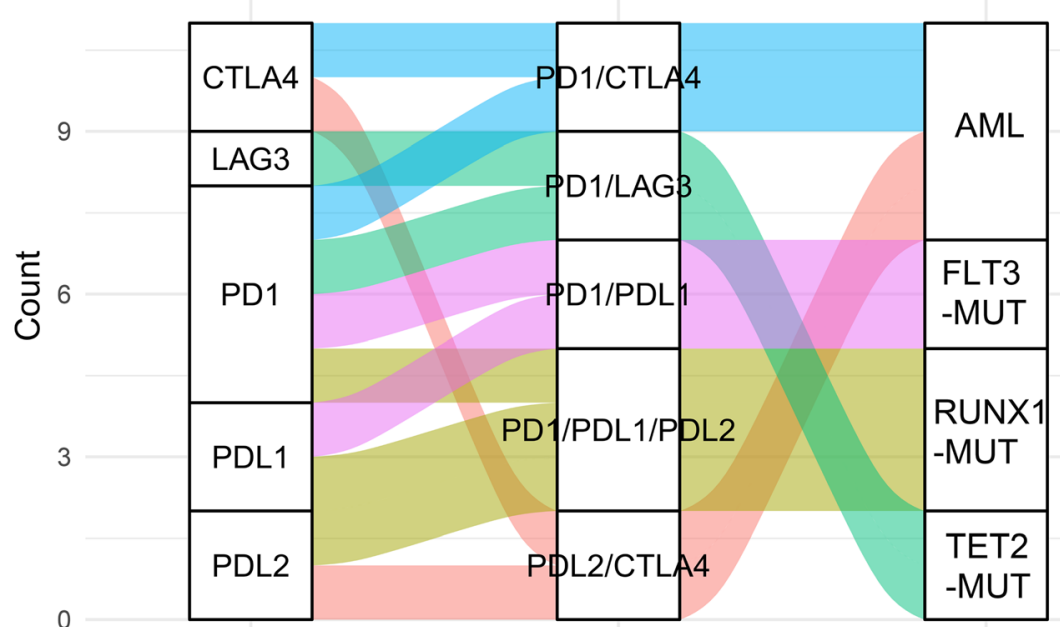

Fig. 2 Co-expression patterns of ICS related to poor OS in AML patients. $\mathbf{a}$, b Comparison of OS curves in AML patients with PD- ${ }^{\text {high }}$, PD-L $1^{\text {high }}$, or PD-L2 ${ }^{\text {high }}$ co-expressed with CTLA-4 ${ }^{\text {low }}$ or CTLA-4 $4^{\text {high }}$ in TCGA group (a) and the validation group $(n=62)(\mathbf{b})$, respectively. $\mathbf{c}$ Comparison of OS curves in AML patients with or without FLT3, RUNX1, or TET2 mutation in TCGA group. mut mutation, wt wildtype. $\mathbf{d}$ Schematic summary of optimal IC combination detection for OS analysis in AML patients with genetic mutations 


\section{Supplementary information}

Supplementary information accompanies this paper at https://doi.org/10. 1186/s13045-020-00853-x.

Additional file 1: Figure S1. Combination of IC detection for OS analysis in patients with AML. A and B: Comparison of OS in patients with high or low expression of PD-1, PD-L1, or PD-L2 co-expressed with high or low CTLA-4 or LAG-3 in the TCGA group (A) and in the validation group $(n=62)(B)$, respectively.

Additional file 2: Figure S2. Comparison of OS in AML patients with high expression PD-1, PD-L1 or PD-L2 co-expressed with high or low $L A G 3$ in the TCGA group (A) and the validation group (B).

Additional file 3: Figure S3. Mutation landscape of the top 10 genes in 176 AML patients in the TCGA database.

Additional file 4: Table S1. Clinical information for the AML patients.

Additional file 5: Table S2. The primers for qRT-PCR.

Additional file 6: Materials and Method

\section{Abbreviations}

AML: Acute myeloid leukemia; BM: Bone marrow; CTLA-4: Cytotoxic Tlymphocyte associated protein 4; FLT3: Fms-related receptor tyrosine kinase 3; IC: Immune checkpoint; ICl: Immune checkpoint inhibitor; LAG3: Lymphocyte activation gene-3; mut: Mutation; PD-1: Programmed cell death 1; PD-L1: Programmed cell death 1 ligand 1; PD-L2: Programmed cell death 1 ligand 2; RUNX1: RUNX family transcription factor 1; TCGA: The Cancer Genome Atlas; TET2: Tetmethylcytosine dioxygenase 2; wt: Wildtype

\section{Acknowledgements}

Not applicable

\section{Authors' contributions}

YQL contributed to the concept development and study design. CTC collected the clinical information, analyzed the data, and wrote the manuscript. CFL performed the experimental research. SHC and CLC contributed to the supervision of the experimental process. CXW, SQW, and YPZ diagnosed and treated the patients and provided clinical bone marrow samples. YQL coordinated the research and helped write the manuscript. All authors read and approved the final manuscript.

\section{Funding}

This study was supported by grants from the National Natural Science Foundation of China (Nos. 81770152, 81570143, and 91642111) and the Guangzhou Science and Technology Project (Nos. 201807010004 and 201803040017).

\section{Availability of data and materials}

All supporting data are included in the manuscript and supplemental files. Additional data are available upon reasonable request to the corresponding author.

\section{Ethics approval and consent to participate}

This study was conducted according to the principals of the Declaration of Helsinki and was approved by the Ethics Committee of Guangzhou First People's Hospital. All participants were provided with written informed consent.

\section{Consent for publication}

Not applicable

\section{Competing interests}

The authors declare that they have no competing interests.

Received: 13 January 2020 Accepted: 3 March 2020

Published online: 03 April 2020

\section{References}

1. Liu D. CAR-T "the living drugs", immune checkpoint inhibitors, and precision medicine: a new era of cancer therapy. J Hematol Oncol. 2019;12:113.
2. Qiu Z, Chen Z, Zhang C, Zhong W. Achievements and futures of immune checkpoint inhibitors in non-small cell lung cancer. Exp Hematol Oncol. 2019;8:19.

3. Huang J, Tan J, Chen Y, Huang S, Xu L, Zhang Y, et al. A skewed distribution and increased PD-1 + V $\beta+$ CD4+/CD8+ T cells in patients with acute myeloid leukemia. J Leukoc Biol. 2019;106(3):725-32.

4. Stahl M, Goldberg AD. Immune checkpoint inhibitors in acute myeloid leukemia: novel combinations and therapeutic targets. Curr Oncol Rep. 2019;21(4):37.

5. Williams P, Basu S, Garcia-Manero G, Hourigan CS, Oetjen KA, Cortes JE, et al. The distribution of T-cell subsets and the expression of immune checkpoint receptors and ligands in patients with newly diagnosed and relapsed acute myeloid leukemia. Cancer. 2019;125(9):1470-81.

6. Mer AS, Lindberg J, Nilsson C, Klevebring D, Wang M, Grönberg H, et al. Expression levels of long non-coding RNAs are prognostic for AML outcome. J Hematol Oncol. 2018;11:52.

7. Smolle MA, Prinz F, Calin GA, Pichler M. Current concepts of non-coding RNA regulation of immune checkpoints in cancer. Mol Aspects Med. 2019; 70:117-26.

8. Tan J, Chen S, Lu Y, Yao D, Xu L, Zhang Y, et al. Higher PD-1 expression concurrent with exhausted CD8+ T cells in patients with de novo acute myeloid leukemia. Chin J Cancer Res. 2017:29(5):463-70.

9. Chen $Y$, Tan J, Huang S, Huang $X$, Huang J, Chen J, et al. Higher frequency of the CTLA-4+ LAG-3+ T-cell subset in patients with newly diagnosed acute myeloid leukemia. Asia Pac J Clin Oncol. 2019. https://doi.org/10. 1111/ajco.13236 [Epub ahead of print].

10. Wu K, Yi M, Qin S, Chu Q, Zheng X, Wu K. The efficacy and safety of combination of PD-1 and CTLA-4 inhibitors: a meta-analysis. Exp Hematol Oncol. 2019;8:26.

11. Daver N, Schlenk RF, Russell NH, Levis MJ. Targeting FLT3 mutations in AML: review of current knowledge and evidence. Leukemia. 2019;33(2):299-312.

12. Gu R, Yang $X$, Wei H. Molecular landscape and targeted therapy of acute myeloid leukemia. Biomark Res. 2018;6:32

13. Zhao J, Song Y, Liu D. Gilteritinib: a novel FLT3 inhibitor for acute myeloid leukemia. Biomark Res. 2019;7:19.

\section{Publisher's Note}

Springer Nature remains neutral with regard to jurisdictional claims in published maps and institutional affiliations.

\section{Ready to submit your research? Choose BMC and benefit from:}

- fast, convenient online submission

- thorough peer review by experienced researchers in your field

- rapid publication on acceptance

- support for research data, including large and complex data types

- gold Open Access which fosters wider collaboration and increased citations

- maximum visibility for your research: over $100 \mathrm{M}$ website views per year

At $\mathrm{BMC}$, research is always in progress.

Learn more biomedcentral.com/submissions 